\title{
Tonic neuromodulation of the inspiratory rhythm generator
}

\author{
Fernando Peña-Ortega* \\ Departamento de Neurobiología del Desarrollo y Neurofisiología, Instituto de Neurobiología, UNAM-Campus Juriquilla, Querétaro, Mexico
}

\author{
Edited by: \\ Francisco Fernandez De-Miguel, \\ Universidad Nacional Autónoma de \\ México, Mexico \\ Reviewed by: \\ Francisco Fernandez De-Miguel, \\ Universidad Nacional Autónoma de \\ México, Mexico \\ Jaime Eugenin, Universidad de \\ Santiago de Chile, Chile \\ Jan Marino Ramirez, University of \\ Washington, USA

\section{*Correspondence:} \\ Fernando Peña-Ortega, \\ Departamento de Neurobiología del \\ Desarrollo y Neurofisiología, Instituto \\ de Neurobiología, Universidad \\ Nacional Autónoma de México, \\ Boulevard Juriquilla 3001, Querétaro \\ 76230, México. \\ e-mail: jfpena@unam.mx
}

The generation of neural network dynamics relies on the interactions between the intrinsic and synaptic properties of their neural components. Moreover, neuromodulators allow networks to change these properties and adjust their activity to specific challenges. Endogenous continuous ("tonic") neuromodulation can regulate and sometimes be indispensible for networks to produce basal activity. This seems to be the case for the inspiratory rhythm generator located in the pre-Bötzinger complex (preBötC). This neural network is necessary and sufficient for generating inspiratory rhythms. The preBötC produces normal respiratory activity (eupnea) as well as sighs under normoxic conditions, and it generates gasping under hypoxic conditions after a reconfiguration process. The reconfiguration leading to gasping generation involves changes of synaptic and intrinsic properties that can be mediated by several neuromodulators. Over the past years, it has been shown that endogenous continuous neuromodulation of the preBötC may involve the continuous action of amines and peptides on extrasynaptic receptors. I will summarize the findings supporting the role of endogenous continuous neuromodulation in the generation and regulation of different inspiratory rhythms, exploring the possibility that these neuromodulatory actions involve extrasynaptic receptors along with evidence of glial modulation of preBötC activity.

Keywords: endogenous neuromodulation, glia, network activity, pacemaker neurons, reconfiguration, extrasynaptic

\section{INTRODUCTION}

Neural network activity relies on the interactions between intrinsic and synaptic properties (Ramirez et al., 2004; Marder and Bucher, 2007; Peña, 2009). Here, I will consider "synaptic properties" as those provided by fast neurotransmission among neurons and "neuromodulation" as the slower changes in cellular and synaptic properties mediated by metabotropic receptors (Katz, 1998). Neuromodulators regulate the activity of networks, allowing their adaptation to different demands or even conditioning their basal activity (Katz, 1998; Tryba et al., 2006; Peña, 2009). This issue has been studied in the inspiratory rhythm generator, the preBötzinger complex (preBötC), which generates the inspiratory commands that control the diaphragm (Smith et al., 1991; Feldman and Del Negro, 2006; Schwarzacher et al., 2011). Breathing is eliminated by lesioning or inactivating the preBötC (Ramirez et al., 1998; Wenninger et al., 2004), whereas the isolated preBötC is still able to generate the inspiratory rhythms in a brainstem slice preparation (Smith et al., 1991; Lieske et al., 2000; Peña et al., 2008; Armstrong et al., 2010) or even in preBötC islands (Ramírez-Jarquín et al., 2012).

In slices, the preBötC generates three distinct activity patterns that correspond to distinct forms of breathing: normal respiratory activity (eupnea), sighs, and gasps (Lieske et al., 2000). Gasping is generated during hypoxia as a "last-resort" respiratory effort to autoresuscitate (Gozal et al., 2002; Fewell et al., 2007; ZavalaTecuapetla et al., 2008). Interestingly, babies that die from SIDS have a reduction in gasping generation and inefficient autoresuscitation (Poets et al., 1999; Sridhar et al., 2003). The preBötC contains several types of neurons, including expiratory, inspiratory, and postinspiratory neurons (Lieske et al., 2000) that interact through fast synaptic transmission (Greer et al., 1991; Funk et al., 1993; Shao and Feldman, 1997; Ren and Greer, 2006) to produce the inspiratory rhythms. Among the inspiratory preBötC neurons, a group of respiratory pacemaker neurons has been detected that plays a major role in rhythm generation (Thoby-Brisson and Ramirez, 2001; Peña et al., 2004; Del Negro et al., 2005; Peña and Aguileta, 2007). Characterization of these neurons revealed at least two types (Thoby-Brisson and Ramirez, 2001; Peña et al., 2004; Del Negro et al., 2005; Peña and Aguileta, 2007; Peña, 2008), one that generates bursts via a $\mathrm{Ca}^{2+}$-activated cationic current $\left(I_{\mathrm{CAN}}\right)$ and the other that relies on the persistent $\mathrm{Na}^{+}$current $\left(I_{\mathrm{NaP}}\right.$; Peña et al., 2004; Del Negro et al., 2005; Peña and Aguileta, 2007; Peña, 2008). Both types of pacemakers need to be inhibited to abolish rhythmogenesis under normoxia, both in vitro and in vivo (Peña et al., 2004; Peña and Ramirez, 2005; Tryba et al., 2006). In contrast, during hypoxic conditions, gasping generation critically relies on the activity of $I_{\mathrm{NaP}}$-dependent (hypoxia-resistant) pacemaker neurons (Peña et al., 2004; Tryba et al., 2006; Peña and Aguileta, 2007). In addition to these mechanisms, the specific contribution of intrinsic and synaptic properties to rhythmogenesis depends on the neuromodulatory context. When applied exogenously, several neuromodulators modify the generation of the rhythmic activity by the preBötC (Doi and Ramirez, 2008). Moreover, several of these neuromodulators maintain a continuous endogenous modulation that, in some cases, is indispensable for rhythm generation (Peña and Ramirez, 2002, 2004; Tryba et al., 2006; Viemari et al., 2011; Ramírez-Jarquín et al., 2012). This continuous modulation, synonymous with "tonic neuromodulation," 
is maintained by continuous release of neuromodulators, mainly from tonic active neurons and glial cells (Hülsmann et al., 2000; Ptak et al., 2009).

\section{CONTINUOUS ("TONIC") NEUROMODULATION OF THE preBötC}

The actions of different neuromodulators on the inspiratory rhythm generator, including amines and peptides, were recently reviewed (Ballanyi, 2004; Peña and García, 2006; Doi and Ramirez, 2008; Peña, 2009). Therefore, I will focus on the evidence of endogenous continuous neuromodulations of the preBötC. In the CNS, several neuromodulators can be continuously released and act both at synaptic and extrasynaptic levels to regulate network function (Vizi et al., 2010). Extrasynaptic transmission was originally discovered for several monoamines that regulate the release of other neuromodulators and neurotransmitters despite the lack of synaptic contact between the two terminals (Vizi et al., 2010). In fact, the majority of monoaminergic and peptidergic neurons fail to make synaptic contacts and instead, they act on extrasynaptic receptors (Descarries and Mechawar, 2000; Vizi et al., 2010). Such neuromodulators are preferentially, but not exclusively, accumulated in large, dense-core vesicles, and they require a strong depolarization or high frequency stimulation to be released (Torrealba and Carrasco, 2004; De-Miguel and Trueta, 2005; Vizi et al., 2010). The fact that several neuromodulators, such as serotonin and adenosine, have been detected in the extracellular space of the preBötC by means of microdialysis (Richter et al., 1999), which detects neurotransmitters and neuromodulators that escaped the synaptic cleft (Peña and Tapia, 1999, 2000), suggests that they can reach extrasynaptic receptors and continuously modulate the preBötC. The extracellular concentration of these neuromodulators changes depending on the state of the network (i.e., hypoxia; Richter et al., 1999; Hehre et al., 2008) indicating that such continuous modulation adjusts the preBötC activity to fit particular demands. Next, I will present a catalog of neuromodulators that maintain a continuous neuromodulation of the preBötC, and discuss the possible involvement of extrasynaptic receptors or glial cells in this modulation. It is important to consider that respiratory rhythmogenesis is studied in a variety of experimental conditions ranging from behaving animals to preBötC islands (RamírezJarquín et al., 2012). Thus, in most cases, the pharmacological manipulations could affect different respiratory circuits besides the preBötC (Zavala-Tecuapetla et al., 2008; Ramírez-Jarquín et al., 2012).

\section{ADENOSINE}

Adenosine is an inhibitory neuromodulator of the preBötC (Schmidt et al., 1995; Herlenius and Lagercrantz, 1999; Wilken et al., 2000; Huxtable et al., 2009) that can be directly released from neurons and glia or that can be extracellularly produced by the degradation of released ATP (Martín et al., 2007; Cunha, 2008; Zwicker et al., 2011). Ambient adenosine can exert its effects by diffusing far away from the release sites (Cunha, 2008; Vizi et al., 2010). An adenosinergic continuous modulation of the preBötC of mice has been evidenced by blocking adenosine-receptors with the non-selective, adenosine-receptor antagonist aminophylline (Wilken et al., 2000), which increases the frequency and amplitude of inspiratory rhythm in slices. This effect is similar to blocking the type 1 (A1) adenosine-receptor in rats with the specific antagonist DPCPX (Huxtable et al., 2009) These increases have also been observed in the brainstem-spinal cord preparation (also called the "en bloc") of rats (Herlenius and Lagercrantz, 1999) and in cats in vivo (Schmidt et al., 1995), where levels of adenosine increase in hypoxia (Richter et al., 1999), contributing to the respiratory depression observed during this condition. In fact, blocking A1receptors attenuates hypoxia-induced breathing in the en bloc of rats (Kawai et al., 1995). Thus, it has been suggested that adenosine antagonists can be useful for the treatment of several respiratory dysfunctions (Mathew, 2011).

\section{ATP}

ATP excites the preBötC in vitro in rats (Huxtable et al., 2009; Zwicker et al., 2011) through the activation of P2Y-receptors (Lorier et al., 2007; Huxtable et al., 2009). Interestingly, blockade of endogenous activation of $\mathrm{P} 2$-receptors with suramin reduced inspiratory frequency in the slice preparation, while $\mathrm{Cu}^{2+}$, an allosteric modulator of purinergic receptors, produced the opposite effect (Lorier et al., 2007, 2008). ATP is released during hypoxia, and blocking its tonic action on P2-receptors increases the hypoxia-induced slowing of the respiratory rhythm, suggesting that ATP is involved in maintaining respiration in hypoxia in rats (Gourine et al., 2005). Interestingly, the excitatory effect of exogenous ATP on the preBötC is precluded when glial cells are inhibited (Huxtable et al., 2009).

\section{ACETYLCHOLINE}

Acetylcholine $(\mathrm{ACh})$ is another neuromodulator that tonically regulates preBötC activity in rats and mice (Shao and Feldman, 2009). Application of the acetylcholinesterase inhibitor physostigmine increases the frequency of rhythmic respiratory activity in the slice preparation involving the type-3-muscarinic and $\alpha 4 \beta 2$-nicotinic receptors in rats and mice, respectively (Shao and Feldman, 2005; Shao et al., 2008). Similarly, blockade of muscarinic-receptors with atropine reduces the amplitude and frequency of the respiratory rhythm in the en bloc from mice (Coddou et al., 2009). In the lamprey en bloc, physostigmine increases the respiratory frequency, while the nicotinic antagonists $\mathrm{D}$-tubocurarine or bungarotoxin reduces it (Mutolo et al., 2011).

\section{NORADRENALINE}

Pre-Bötzinger complex activity is modulated by endogenous noradrenaline released from the $\mathrm{A} 5, \mathrm{~A} 6, \mathrm{~A} 1 \mathrm{C} 1$, and $\mathrm{A} 2 \mathrm{C} 2$ nuclei in rats and mice (Hilaire et al., 2004; Viemari, 2008). This continuous modulation involves activation of $\alpha$-2-adrenoreceptors, since its blockade with yohimbine, piperoxane, or phentolamine decreases respiratory frequency in the en bloc in rats and mice (Errchidi et al., 1990; Zanella et al., 2006; Fujii and Arata, 2010) and abolishes gasping generation in slices from mice (Viemari et al., 2011). Accordingly, decreasing the extracellular noradrenaline concentration with pargyline, desipramine, or tyrosine increases the frequency of the rhythm, while methyltyrosine, an inhibitor of noradrenaline biosynthesis, increases the en bloc respiratory frequency in rats and mice (Errchidi et al., 1990; Zanella et al., 2006). There is some evidence of a continuous modulation of the preBötC by histamine and dopamine. Thus, the histamine-type1-receptor antagonist, pyrilamine, reduces the en bloc respiratory 
frequency and attenuates respiratory depression in hypoxia in mice (Dutschmann et al., 2003), while the dopamine-type-1-receptor antagonist SCH-23390 slows the respiratory rhythm of cats in vivo (Lalley, 2004, 2005).

\section{SEROTONIN}

The preBötC is modulated by 5 -hydroxytryptamine (5-HT), which produces an excitatory effect mediated by 5-HT2-receptors and an inhibitory effect mediated by 5 -HT1-receptors (Schwarzacher et al., 2002). The main source of 5-HT is the raphe nuclei (Richerson, 2004), whose projections can or cannot make synaptic contacts with their targets throughout the brain (Kosofsky and Molliver, 1987). In the preBötC, increasing the extracellular concentration of 5-HT with 5-HT-uptake inhibitors leads to an increase of respiratory activity in the en bloc from rats (Di Pasquale et al., 1994). In contrast, blocking 5-HT-receptors with the non-specific antagonist methysergide abolishes rhythmogenesis in the en bloc and in slices from rats (Di Pasquale et al., 1994; Ptak et al., 2009). In these preparations, excitation of raphe neurons increases the frequency of the respiratory rhythm mediated by the activation of 5-HT2-receptors (Al-Zubaidy et al., 1996; Ptak et al., 2009). Accordingly, blocking either 5-HT2Breceptors (Günther et al., 2006), 5-HT2C-receptors (Ptak et al., 2009), or 5-HT2A receptors (Peña and Ramirez, 2002; Ptak et al., 2009) reduces the respiratory rhythm frequency and its regularity in slices from rats and mice. Such findings have been corroborated for 5-HT2A- and 5-HT2C-receptors in situ in rats (Ptak et al., 2009). Interestingly, low micromolar concentrations of 5HT induce bursting activity in non-bursting preBötC neurons (Ptak et al., 2009), while blockade of 5-HT2A receptors abolishes the intrinsic bursting of the $I_{\mathrm{NaP}}$-dependent (hypoxia-resistant) pacemaker neurons (Peña and Ramirez, 2002; Tryba et al., 2006). Consequently, blockade of 5-HT2A receptors inhibits gasping generation in slices from mice (Tryba et al., 2006) and in situ in rats (Bale and Solomon, 2010). These findings may have clinical relevance, since it has been hypothesized that a deficiency of the medullary 5-HT network is a potential cause of SIDS (Kinney et al., 2001).

\section{PEPTIDES}

Several neuropeptides may exert a continuous regulation of the preBötC. Neuropeptides are typical non-synaptic transmitters, which are released extrasynaptically (Torrealba and Carrasco, 2004; Wotjak et al., 2008). Blocking the endogenous activation of the opioid-receptors with naloxone increases the respiratory output in cats (Lawson et al., 1979) and reduces hypoxia-induced respiratory depression in rats (Schlenker and Inamdar, 1995). In mice, blocking endogenous activation of somatostatin-receptors increases the respiratory rhythm frequency and reduces its regularity, both in slices and in vivo (Ramírez-Jarquín et al., 2012). Moreover, blockade of somatostatin-receptors, specifically subtype 2, prevents the reconfiguration of the preBötC during hypoxia in vitro and reduces gasping generation and autoresuscitation in vivo (Ramírez-Jarquín et al., 2012). In contrast, substance-P maintains an excitatory continuous modulation on the preBötC in rats and mice (Ptak et al., 2009; Doi and Ramirez, 2010). Blockade of the substance-P receptor (NK1) with SR 140333 or spantide inhibits rhythmogenesis in vitro and in situ in mice and rats, respectively (Telgkamp et al., 2002; Ptak et al., 2009). Interestingly, in mice, inhibition of respiratory activity with NK1 antagonists has no significant respiratory effect when the levels of 5-HT or noradrenaline are increased by stimulating the raphe magnus or locus coeruleus, respectively (Doi and Ramirez, 2010), indicating that the action of substance-P might be influenced by the neuromodulatory state of the network (Doi and Ramirez, 2010).

\section{POSSIBLE REGULATION OF THE preBötC BY GABA AND GLUTAMATE ACTING ON EXTRASYNAPTIC RECEPTORS}

Glutamatergic and GABAergic neurons were thought to release their transmitters exclusively at synapses, where they mediate the classical "fast synaptic transmission" (Vizi et al., 2010). However, it has been shown that ambient GABA and glutamate can also tonically activate high-affinity, extrasynaptic receptors, suggesting their spill-over from synaptic boutons, mediating a slower synaptic transmission (Semyanov et al., 2004; Farrant and Nusser, 2005; Aghajanian, 2009). Extrasynaptic GABA A inhibition can modulate the generation of hippocampal fast rhythms (Scanziani, 2000; Towers et al., 2004; Mann and Mody, 2010; Papatheodoropoulos and Koniaris, 2011), and it is likely that such modulation also occurs in the preBötC, where increasing the extracellular concentration of GABA, by inhibiting its uptake with nipecotic acid, decreases the respiratory frequency (Ren and Greer, 2006). The presence of delta-subunit-containing$\mathrm{GABA}_{\mathrm{A}}$-receptors, which are mainly extrasynaptic (Nusser et al., 1998; Adkins et al., 2001; Brown et al., 2002) suggests a tonic GABAergic control of the preBötC. For instance, the application of the $\mathrm{GABA}_{\mathrm{A}}$-receptor agonist THIP, which preferentially activates extrasynaptic $\mathrm{GABA}_{\mathrm{A}}$-receptors containing delta-subunits (Nusser et al., 1998; Adkins et al., 2001; Brown et al., 2002), hyperpolarizes respiratory neurons and reduces the frequency of the respiratory rhythm (Shao and Feldman, 1997). Neurosteroids, which also target delta-containing, extrasynaptic $\mathrm{GABA}_{\mathrm{A}}$-receptors (Stell et al., 2003; Belelli and Lambert, 2005; Scimemi et al., 2006), modulate $\mathrm{GABA}_{\mathrm{A}}$-receptor-mediated hyperpolarization of respiratory neurons and the inhibition of rhythmogenesis in slices (Ren and Greer, 2006).

Ambient glutamate can also activate extrasynaptic, NR2Bsubunit-containing, NMDA-receptors and modulate neural network activity (Lambe and Aghajanian, 2006, 2007; Aghajanian, 2009). It is likely that extrasynaptic, NMDA-receptor-mediated excitation is also present in the preBötC, where inhibition of glutamate uptake with dihydrokainate increases rhythmogenesis (Greer et al., 1991; Funk et al., 1993). Dihydrokainate can also restore rhythmogenesis in substance-P-depleted slices, in which capsaicin abolishes rhythm generation (Morgado-Valle and Feldman, 2004). Similarly, releasing NMDA-receptors from their $\mathrm{Mg}^{2+}$-blockade restores rhythmogenesis in slices where the rhythm is abolished by AMPA-receptor blockade (Morgado-Valle and Feldman, 2007). This evidence supports the notion that a tone of extracellular glutamate can participate in rhythmogenesis. Furthermore, the presence of the NR2B-receptor has been extensively documented in the preBötC (Watanabe et al., 1994; Paarmann et al., 2000, 2005; Liu and Wong-Riley, 2010). 


\section{GLIAL MODULATION OF THE preBötC}

Glial cells are integral functional elements of neural networks, since it is argued that they can respond to and regulate neuronal activity (Araque and Navarrete, 2010). The respiratory network is not an exception (Gourine et al., 2010). Glial cells can sense preBötC activity, and a portion of them display a phase-locked rhythmic activity (Schnell et al., 2011). Moreover, glial cells are essential for rhythmogenesis, since both fluoroacetate, which selectively blocks the glial Krebs cycle, and methionine-sulfoximine, which blocks glutamine synthetase (Hülsmann et al., 2000; Young et al., 2005; Huxtable et al., 2010), inhibit rhythmic respiratory burst activity in slices. In these conditions, addition of isocitrate or glutamine restores the rhythmic network activity (Hülsmann et al., 2000). Accordingly, methionine-sulfoximine-treated pups displayed a reduced breathing frequency and a reduced responsiveness to hypercapnia (Young et al., 2005). Moreover, glial cells are required not only for maintaining rhythm generation but also for the response of the preBötC to neuromodulators or to metabolic demands (Gourine et al., 2010). For instance, fluoroacetate and methioninesulfoximine reduce preBötC responsiveness to ATP (Huxtable et al., 2010), and preBötC glial cells can respond to preBötC neuromodulators including 5-HT and substance-P (Härtel et al., 2009).

\section{REFERENCES}

Adkins, C. E., Pillai, G. V., Kerby, J., Bonnert, T. P., Haldon, C., McKernan, R. M., Gonzalez, J. E., Oades, K., Whiting, P. J., and Simpson, P. B. (2001). Alpha4beta3delta GABAA receptors characterized by fluorescence resonance energy transfer-derived measurements of membrane potential. J. Biol. Chem. 276, 38934-38939.

Aghajanian, G. K. (2009). Modeling "psychosis" in vitro by inducing disordered neuronal network activity in cortical brain slices. Psychopharmacology (Berl.) 206, 575-585.

Al-Zubaidy, Z. A., Erickson, R. L., and Greer, J. J. (1996). Serotonergic and noradrenergic effects on respiratory neural discharge in the medullary slice preparation of neonatal rats. Pflugers Arch. 431, 942-949.

Araque, A., and Navarrete, M. (2010). Glial cells in neuronal network function. Philos. Trans. R. Soc. Lond. B. Biol. Sci. 365, 2375-2381.

Armstrong, G. A., López-Guerrero, J. J., Dawson-Scully, K., Peña, F., and Robertson, R. M. (2010). Inhibition of protein kinase $G$ activity protects neonatal mouse respiratory network from hyperthermic and hypoxic stress. Brain Res. 1311, 64-72.

Bale, T. A., and Solomon, I. C. (2010). Influence of 5-HT2A receptor blockade on phrenic nerve discharge at three levels of extracellular $\mathrm{K}+$ in arterially-perfused adult rat. $A d v$. Exp. Med. Biol. 669, 139-142.
Ballanyi, K. (2004). Neuromodulation of the perinatal respiratory network. Curr. Neuropharmacol. 2, 221-243.

Belelli, D., and Lambert, J. J. (2005). Neurosteroids, endogenous regulators of the GABAA receptor. Nat. Rev. Neurosci. 7, 565-575.

Brown, N., Kerby, J., Bonnert, T. P., Whiting, P. J., and Wafford, K. A. (2002). Pharmacological characterization of a novel cell line expressing human alpha 4beta 3delta GABAA. receptors. Br. J. Pharmacol. 136, 965-974.

Coddou, C., Bravo, E., and Eugenín, J. (2009). Alterations in cholinergic sensitivity of respiratory neurons induced by pre-natal nicotine, a mechanism for respiratory dysfunction in neonatal mice. Philos. Trans. R. Soc. Lond. B. Biol. Sci. 364, 2527-2535.

Cunha, R. A. (2008). Different cellular sources and different roles of adenosine, $\mathrm{A} 1$ receptor-mediated inhibition through astrocytic-driven volume transmission and synapserestricted $\mathrm{A} 2 \mathrm{~A}$ receptor-mediated facilitation of plasticity. Neurochem. Int. 52, 65-72.

Del Negro, C. A., Morgado-Valle, C., Hayes, J. A., Mackay, D. D., Pace, R. W., Crowder, E. A., and Feldman, J. L. (2005). Sodium and calcium current-mediated pacemaker neurons and rhythmogenesis. J. Neurosci. $25,446-453$.

I conclude that continuous neuromodulation exerts a powerful influence on the preBötC; to the extent that, in some cases, it is necessary for rhythm generation. Continuous neuromodulation tunes the excitability of the preBötC to respond to different demands and also determines the weight of specific neuronal types or specific synaptic interactions in the generation of network dynamics. This property could allow the preBötC to adopt an infinite number of conformations based on the same circuit (neural units and connections). Moreover, the evidence that one neuromodulation is determined by tonic control exerted by other neuromodulators, supports the notion that the intrinsic and synaptic properties of the preBötC are not fixed, but can change in a state-dependent manner. The levels of modulation in the preBötC would determine the availability of neural properties (intrinsic, synaptic, or both) that can participate in network dynamics or are susceptible to subsequent neuromodulation.

\section{ACKNOWLEDGMENTS}

I would like to thank Dorothy Pless for reviewing the English version of this paper. I also thank José Rodolfo Fernandez and Arturo Franco for technical assistance. The research in my group is sponsored by grants (to F P-O) from DGAPA IB200212, CONACyT 151261, 181323 and from the Alzheimer's Association NIRG-11-205443.

De-Miguel, F. F., and Trueta, C. (2005). Synaptic and extrasynaptic secretion of serotonin. Cell. Mol. Neurobiol. 25, 297-312.

Descarries, L., and Mechawar, N. (2000). Ultrastructural evidence for diffuse transmission by monoamine and acetylcholine neurons of the central nervous system. Prog. Brain Res. 125, 27-47.

Di Pasquale, E., Monteau, R., and Hilaire, G. (1994). Endogenous serotonin modulates the fetal respiratory rhythm, an in vitro study in the rat. Brain Res. Dev. Brain Res. 80 222-232.

Doi, A., and Ramirez, J. M. (2008). Neuromodulation and the orchestration of the respiratory rhythm. Respir. Physiol. Neurobiol. 164, 96-104.

Doi, A., and Ramirez, J. M. (2010). Statedependent interactions between excitatory neuromodulators in the neuronal control of breathing. $J$. Neurosci. 30, 8251-8262.

Dutschmann, M., Bischoff, A. M., Büsselberg, D., and Richter, D. W. (2003). Histaminergic modulation of the intact respiratory network of adult mice. Pflugers Arch. 445, 570-576.

Errchidi, S., Hilaire, G., and Monteau, R. (1990). Permanent release of noradrenaline modulates respiratory frequency in the newborn rat, an in vitro study. J. Physiol. (Lond.) 429, 497-510.

Farrant, M., and Nusser, Z. (2005). Variations on an inhibitory theme, phasic and tonic activation of GABA A). receptors. Nat. Rev. Neurosci. 6, 215-229.

Feldman, J. L., and Del Negro, C. A. (2006). Looking for inspiration, new perspectives on respiratory rhythm. Nat. Rev. Neurosci. 7, 232-242.

Fewell, J. E., Zhang, C., and Gillis, A. M. (2007). Influence of adenosine A1receptor blockade and vagotomy on the gasping and heart rate response to hypoxia in rats during early postnatal maturation. J. Appl. Physiol. 103, 1234-1241.

Fujii, M., and Arata, A. (2010). Adrenaline modulates on the respiratory network development. Adv. Exp. Med. Biol. 669, 25-28.

Funk, G. D., Smith, J. C., and Feldman, J. L. (1993). Generation and transmission of respiratory oscillations in medullary slices, role of excitatory amino acids. J. Neurophysiol. 70, 1497-1515.

Gourine, A. V., Kasymov, V., Marina, N., Tang, F., Figueiredo, M. F., Lane, S., Teschemacher, A. G., Spyer, K. M., Deisseroth, K., and Kasparov, S. (2010). Astrocytes control breathing through $\mathrm{pH}$-dependent release of ATP. Science 329, 571-575.

Gourine, A. V., Llaudet, E., Dale, N., and Spyer, K. M. (2005). Release of ATP in the ventral medulla during hypoxia in rats, role in hypoxic ventilatory response. J. Neurosci. 25, 1211-1218. 
Gozal, D., Gozal, E., Reeves, S. R., and Lipton, A. J. (2002). Gasping and autoresuscitation in the developing rat, effect of antecedent intermittent hypoxia. J. Appl. Physiol. 92, 1141-1144.

Greer, J. J., Smith, J. C., and Feldman, J. L. (1991). Role of excitatory amino acids in the generation and transmission of respiratory drive in neonatal rat. J. Physiol. (Lond.) 437, 727-749.

Günther, S., Maroteaux, L., and Schwarzacher, S. W. (2006). Endogenous 5-HT2B receptor activation regulates neonatal respiratory activity in vitro. J. Neurobiol. 66, 949-961.

Härtel, K., Schnell, C., and Hülsmann, S. (2009). Astrocytic calcium signals induced by neuromodulators via functional metabotropic receptors in the ventral respiratory group of neonatal mice. Glia 57, 815-827.

Hehre, D. A., Devia, C. J., Bancalari, E., and Suguihara, C. (2008). Brainstem amino acid neurotransmitters and ventilatory response to hypoxia in piglets. Pediatr. Res. 63, 46-50.

Herlenius, E., and Lagercrantz, H. (1999). Adenosinergic modulation of respiratory neurons in the neonatal rat brainstem in vitro. J. Physiol. (Lond.) 518, 159-172.

Hilaire, G., Viemari, J. C., Coulon, P., Simonneau, M., and Bévengut, M. (2004). Modulation of the respiratory rhythm generator by the pontine noradrenergic A5 and A6 groups in rodents. Respir. Physiol. Neurobiol. 143, 187-197.

Hülsmann, S., Oku, Y., Zhang, W., and Richter, D. W. (2000). Metabolic coupling between glia and neurons is necessary for maintaining respiratory activity in transverse medullary slices of neonatal mouse. Eur. J. Neurosci. 12, 856-862.

Huxtable, A. G., Zwicker, J. D., Alvares, T. S., Ruangkittisakul, A., Fang, X., Hahn, L. B., Posse de Chaves, E., Baker, G. B., Ballanyi, K., and Funk, G. D. (2010). Glia contribute to the purinergic modulation of inspiratory rhythm-generating networks. $J$. Neurosci. 30, 3947-3958.

Huxtable, A. G., Zwicker, J. D., Poon, B. Y., Pagliardini, S., Vrouwe, S. Q., Greer, J. J., and Funk, G. D. (2009). Tripartite purinergic modulation of central respiratory networks during perinatal development, the influence of ATP., ectonucleotidases, and ATP metabolites. J. Neurosci. 29, 14713-14725.

Katz, P. S. (1998). Comparison of extrinsic and intrinsic neuromodulation in two central pattern generator circuits in invertebrates. Exp. Physiol. 83, 281-292.

Kawai, A., Okada, Y., Mückenhoff, K., and Scheid, P. (1995). Theophylline and hypoxic ventilator response in the rat isolated brainstemspinal cord. Respir. Physiol. 100, 25-32.

Kinney, H. C., Filiano, J. J., and White, W. F. (2001). Medullary serotonergic network deficiency in the sudden infant death syndrome, review of a 15-year study of a single dataset. J. Neuropathol. Exp. Neurol. 60, 228-247.

Kosofsky, B. E., and Molliver, M. E. (1987). The serotoninergic innervation of cerebral cortex, different classes of axon terminals arise from dorsal and median raphe nuclei. Synapse 2, 153-168.

Lalley, P. M. (2004). Dopaminel receptor agonists reverse opioid respiratory network depression, increase $\mathrm{CO} 2$ reactivity. Respir. Physiol. Neurobiol. 139, 247-262.

Lalley, P. M. (2005). D1-dopamine receptor blockade slows respiratory rhythm and enhances opioidmediated depression. Respir. Physiol. Neurobiol. 145, 13-22.

Lambe, E. K., and Aghajanian, G. K. (2006). Hallucinogen-induced UP states in the brain slice of rat prefrontal cortex, role of glutamate spillover and NR2B-NMDA receptors. Neuropsychopharmacology 31, 1682-1689.

Lambe, E. K., and Aghajanian, G. K. (2007). Prefrontal cortical network activity, opposite effects of psychedelic hallucinogens and D1/D5 dopamine receptor activation. $\mathrm{Neu}$ roscience 145, 900-910.

Lawson, E. E., Waldrop, T. G., and Eldridge, F. L. (1979). Naloxone enhances respiratory output in cats. J. Appl. Physiol. 47, 1105-1111.

Lieske, S. P., Thoby-Brisson, M., Telgkamp, P., and Ramirez, J. M. (2000). Reconfiguration of the neural network controlling multiple breathing patterns, eupnea, sighs and gasps. Nat. Neurosci. 3, 600-607.

Liu, Q., and Wong-Riley, M. T. (2010). Postnatal development of N-methyl$\mathrm{D}$-aspartate receptor subunits $2 \mathrm{~A}$, $2 \mathrm{~B}, 2 \mathrm{C}, 2 \mathrm{D}$, and $3 \mathrm{~B}$ immunoreactivity in brain stem respiratory nuclei of the rat. Neuroscience 171, 637-654.

Lorier, A. R., Huxtable, A. G., Robinson, D. M., Lipski, J., Housley, G. D., and Funk, G. D. (2007). P2Y1 receptor modulation of the pre-Bötzinger complex inspiratory rhythm generating network in vitro. J. Neurosci. 27, 993-1005.
Lorier, A. R., Lipski, J., Housley, G. D. Greer, J. J., and Funk, G. D. (2008). ATP sensitivity of preBötzinger complex neurones in neonatal rat in vitro, mechanism underlying a $\mathrm{P} 2$ receptor-mediated increase in inspiratory frequency. J. Physiol. (Lond.) 586, 1429-1446.

Mann, E. O., and Mody, I. (2010). Control of hippocampal gamma oscillation frequency by tonic inhibition and excitation of interneurons. Nat. Neurosci. 13, 205-212.

Marder, E., and Bucher, D. (2007). Understanding circuit dynamics using the stomatogastric nervous system of lobsters and crabs. Annu. Rev. Physiol. 69, 291-316.

Martín, E. D., Fernández, M., Perea, G., Pascual, O., Haydon, P. G., Araque, A., and Ceña, V. (2007). Adenosine released by astrocytes contributes to hypoxia-induced modulation of synaptic transmission. Glia 55, 36-45.

Mathew, O. P. (2011). Apnea of prematurity, pathogenesis, and management strategies. J. Perinatol. 31 , 302-310.

Morgado-Valle, C., and Feldman, J. L. (2004). Depletion of substance P and glutamate by capsaicin blocks respiratory rhythm in neonatal rat in vitro. J. Physiol. (Lond.) 555, 783-792.

Morgado-Valle, C., and Feldman, J. L. (2007). NMDA receptors in preBotzinger complex neurons can drive respiratory rhythm independent of AMPA receptors. J. Physiol. (Lond.) 582, 359-368.

Mutolo, D., Cinelli, E., Bongianni, F., and Pantaleo, T. (2011). Identification of a cholinergic modulatory and rhythmogenic mechanism within the lamprey respiratory network. J. Neurosci. 31, 13323-13332.

Nusser, Z., Sieghart, W., and Somogyi, P. (1998). Segregation of different GABAA receptors to synaptic, and extrasynaptic membranes of cerebellar granule cells. J. Neurosci. 18, 1693-1703.

Paarmann, I., Frermann, D., Keller, B. U., and Hollmann, M. (2000). Expression of 15 glutamate receptor subunits and various splice variants in tissue slices and single neurons of brainstem nuclei and potential functional implications. J. Neurochem. 74, 1335-1345.

Paarmann, I., Frermann, D., Keller, B. U., Villmann, C., Breitinger, H. G., and Hollmann, M. (2005). Kinetics and subunit composition of NMDA receptors in respiratoryrelated neurons. J. Neurochem. 93, 812-824.
Papatheodoropoulos, C., and Koniaris, E. (2011). $\alpha 5$ GABAA receptors regulate hippocampal sharp wave-ripple activity in vitro. Neuropharmacology 60, 662-673.

Peña, F. (2008). Contribution of pacemaker neurons to respiratory rhythms generation in vitro. $A d v$. Exp. Med. Biol. 605, 114-118.

Peña, F. (2009). Neuronal network properties underlying the generation of gasping. Clin. Exp. Pharmacol. Physiol. 36, 1218-1228.

Peña, F., and Aguileta, M. A. (2007). Effects of riluzole, and flufenamic acid on eupnea, and gasping of neonatal mice in vivo. Neurosci. Lett. 415, 288-293.

Peña, F., and García, O. (2006). Breathing generation and potential pharmacotherapeutic approaches to central respiratory disorders. Curr. Med. Chem. 13, 2681-2693.

Peña, F., Meza-Andrade, R., Páez-Zayas, V., and González-Marín, M. C. (2008). Gasping generation in developing Swiss-Webster mice in vitro and in vivo. Neurochem. Res. 33, 1492-1500.

Peña, F., Parkis, M. A., Tryba, A. K., and Ramirez, J. M. (2004). Differential contribution of pacemaker properties to the generation of respiratory rhythms during normoxia, and hypoxia. Neuron 43, 105-117.

Peña, F., and Ramirez, J. M. (2002). Endogenous activation of serotonin$2 \mathrm{~A}$ receptors is required for rhythmogenesis in vitro. J. Neurosci. 22, 11055-11064.

Peña, F., and Ramirez, J. M. (2004). Substance P-mediated modulation of pacemaker properties in the mammalian respiratory network. J. Neurosci. 24, 7549-7556.

Peña, F., and Ramirez, J. M. (2005). Hypoxia-induced changes in neuronal network properties. $\mathrm{Mol}$. Neurobiol. 32, 251-283.

Peña, F., and Tapia, R. (1999). Relationships among seizures, extracellular amino acid changes, and neurodegeneration induced by 4 aminopyridine in rat hippocampus: a microdialysis and electrncephalographic study. J. Neurochem. 72, 2006-2014.

Peña, F., and Tapia, R. (2000). Seizures and neurodegeneration induced by 4-aminopyridine in rat hippocampus in vivo: role of glutamate- and GABA-mediated neurotransmission and of ion channels. Neuroscience 101, 547-561.

Poets, C. F., Meny, R. G., Chobanian, M. R., and Bonofiglo, R. E. (1999). Gasping and other 
cardiorespiratory patterns during sudden infant deaths. Pediatr. Res. 45, 350-354.

Ptak, K., Yamanishi, T., Aungst, J., Milescu, L. S., Zhang, R., Richerson, G. B., and Smith, J. C. (2009). Raphé neurons stimulate respiratory circuit activity by multiple mechanisms via endogenously released serotonin and substance P. J. Neurosci. 29, 3720-3737.

Ramirez, J. M., Schwarzacher, S. W., Pierrefiche, O., Olivera, B. M., and Richter, D. W. (1998). Selective lesioning of the cat pre-Bötzinger complex in vivo eliminates breathing but not gasping. J. Physiol. (Lond.) 507, 895-907.

Ramirez, J. M., Tryba, A. K., and Peña, F. (2004). Pacemaker neurons and neuronal networks, an integrative view. Curr. Opin. Neurobiol. 14, 665-674.

Ramírez-Jarquín, J. O., LaraHernández, S., López-Guerrero, J. J., Aguileta, M. A., Rivera-Angulo, A. J., Sampieri, A., Vaca, L., Ordaz, B., and Peña-Ortega, F. (2012). Somatostatin modulates generation of inspiratory rhythms and determines asphyxia survival. Peptides 34, 360-372.

Ren, J., and Greer, J. J. (2006). Neurosteroid modulation of respiratory rhythm in rats during the perinatal period. J. Physiol. (Lond.) 574, 535-546.

Richerson, G. B. (2004). Serotonergic neurons as carbon dioxide sensors that maintain $\mathrm{pH}$ homeostasis. Nat. Rev. Neurosci. 5, 449-461.

Richter, D. W., Schmidt-Garcon, P., Pierrefiche, O., Bischoff, A. M., and Lalley, P. M. (1999). Neurotransmitters and neuromodulators controlling the hypoxic respiratory response in anaesthetized cats. J. Physiol. (Lond.) 514, 567-578.

Scanziani, M. (2000). GABA spillover activates postsynaptic GABA B) receptors to control rhythmic hippocampal activity. Neuron 25, 673-681.

Schlenker, E. H., and Inamdar, S. R. (1995). Effects of naloxone on oxygen consumption and ventilation in awake golden Syrian hamsters. Physiol. Behav. 57, 655-658.

Schmidt, C., Bellingham, M. C., and Richter, D. W. (1995). Adenosinergic modulation of respiratory neurones and hypoxic responses in the anaesthetized cat. J. Physiol. (Lond.) 483, 769-781.

Schnell, C., Fresemann, J., and Hülsmann, S. (2011). Determinants of functional coupling between astrocytes and respira- tory neurons in the pre-Bötzinger complex. PLoS ONE 6, e26309. doi:10.1371/journal.pone.0026309

Schwarzacher, S. W., Pestean, A., Günther, S., and Ballanyi, K. (2002) Serotonergic modulation of respiratory motoneurons and interneurons in brainstem slices of perinatal rats. Neuroscience 115, 1247-1259.

Schwarzacher, S. W., Rüb, U., and Deller, T. (2011). Neuroanatomical characteristics of the human pre-Bötzinger complex and its involvement in neurodegenerative brainstem diseases. Brain 134, 24-35.

Scimemi, A., Andersson, A., Heeroma, J. H., Strandberg, J., Rydenhag, B., McEvoy, A. W., Thom, M., Asztely, F., and Walker, M. C. (2006). Tonic GABAA receptor-mediated currents in human brain. Eur. J. Neurosci. 24, 1157-1160.

Semyanov, A., Walker, M. C., Kullmann, D. M., and Silver, R. A. (2004). Tonically active GABA A receptors, modulating gain and maintaining the tone. Trends Neurosci. 27, 262-269.

Shao, X. M., and Feldman, J. L. (1997). Rhythmogenesis and synaptic inhibition of expiratory neurons in preBötzinger complex, differential roles of glycinergic and GABAergic neural transmission. J. Neurophysiol. 77, 1853-1860.

Shao, X. M., and Feldman, J. L. (2005). Cholinergic neurotransmission in the preBötzinger Complex modulates excitability of inspiratory neurons and regulates respiratory rhythm. Neuroscience 130, 1069-1081.

Shao, X. M., and Feldman, J. L. (2009). Central cholinergic regulation of respiration, nicotinic receptors. Acta Pharmacol. Sin. 30, 761-770.

Shao, X. M., Tan, W., Xiu, J., Puskar, N., Fonck, C., Lester, H. A., and Feldman, J. L. (2008). Alpha4* nicotinic receptors in preBotzinger complex mediate cholinergic/nicotinic modulation of respiratory rhythm. J. Neurosci. 28, 519-528.

Smith, J. C., Ellenberger, H. H., Ballanyi, K., Richter, D. W., and Feldman, J. L. (1991). Pre-Bötzinger complex: a brainstem region that may generate respiratory rhythm in mammals. Science 254, 726-729.

Sridhar, R., Thach, B. T., Kelly, D. H., and Henslee, J. A. (2003). Characterization of successful and failed autoresuscitation in human infants, including those dying of SIDS. Pediatr. Pulmonol. 36, 113-122.

Stell, B. M., Brickley, S. G., Tang, C. Y., Farrant, M., and Mody, I.
(2003). Neuroactive steroids reduce neuronal excitability by selectively enhancing tonic inhibition mediated by delta subunit-containing GABAA receptors. Proc. Natl. Acad. Sci. U.S.A. 100, 14439-14444.

Telgkamp, P., Cao, Y. Q., Basbaum, A. I., and Ramirez, J. M. (2002). Long-term deprivation of substance $\mathrm{P}$ in PPT-A mutant mice alters the anoxic response of the isolated respiratory network. J. Neurophysiol. 88, 206-213.

Thoby-Brisson, M., and Ramirez, J. M. (2001). Identification of two types of inspiratory pacemaker neurons in the isolated respiratory neural network of mice. J. Neurophysiol. 86, 104-112.

Torrealba, F., and Carrasco, M. A. (2004). A review on electron microscopy and neurotransmitter systems. Brain Res. Brain Res. Rev. 47, 5-17.

Towers, S. K., Gloveli, T., Traub, R. D. Driver, J. E., Engel, D., Fradley, R., Rosahl, T. W., Maubach, K., Buhl, E. H., and Whittington, M. A. (2004). Alpha 5 subunit-containing GABAA receptors affect the dynamic range of mouse hippocampal kainateinduced gamma frequency oscillations in vitro. J. Physiol. (Lond.) 559 721-728.

Tryba, A. K., Peña, F., and Ramirez, J. M. (2006). Gasping activity in vitro, a rhythm dependent on 5-HT2A receptors. J. Neurosci. 26, 2623-2634.

Viemari, J. C. (2008). Noradrenergic modulation of the respiratory neural network. Respir. Physiol. Neurobiol. 164, 123-130.

Viemari, J. C., Garcia, A. J. III, Doi, A., and Ramirez, J. M. (2011). Activation of alpha-2 noradrenergic receptors is critical for the generation of fictive eupnea and fictive gasping inspiratory activities in mammals in vitro. Eur. J. Neurosci. 33, 2228-2237.

Vizi, E. S., Fekete, A., Karoly, R. and Mike, A. (2010). Non-synaptic receptors and transporters involved in brain functions and targets of drug treatment. Br. J. Pharmacol. 160, 785-809.

Watanabe, M., Mishina, M., and Inoue, Y. (1994). Distinct distributions of five NMDA receptor channel subunit mRNAs in the brainstem. J. Comp. Neurol. 343, 520-531.

Wenninger, J. M., Pan, L. G., Klum, L., Leekley, T., Bastastic, J., Hodges, M. R., Feroah, T. R., Davis, S., and Forster, H. V. (2004). Large lesions in the pre-Bötzinger complex area eliminate eupneic respiratory rhythm in awake goats. J. Appl. Physiol. 97, 1629-1636.

Wilken, B., Ramirez, J. M., Hanefeld, F., and Richter, D. W. (2000). Aminophylline modulation of the mouse respiratory network changes during postnatal maturation. J. Appl. Physiol. 89, 2015-2022.

Wotjak, C. T., Landgraf, R., and Engelmann, M. (2008). Listening to neuropeptides by microdialysis, echoes and new sounds? Pharmacol. Biochem. Behav. 90, 125-134.

Young, J. K., Dreshaj, I. A., Wilson, C. G., Martin, R. J., Zaidi, S. I., and Haxhiu, M. A. (2005). An astrocyte toxin influences the pattern of breathing and the ventilatory response to hypercapnia in neonatal rats. Respir. Physiol. Neurobiol. 147, 19-30.

Zanella, S., Roux, J. C., Viemari, J. C., and Hilaire, G. (2006). Possible modulation of the mouse respiratory rhythm generator by $\mathrm{Al} / \mathrm{Cl}$ neurones. Respir. Physiol. Neurobiol. 153, 126-138.

Zavala-Tecuapetla, C., Aguileta, M. A., Lopez-Guerrero, J. J., GonzálezMarín, M. C., and Peña, F. (2008). Calcium-activated potassium currents differentially modulate rhythmogenesis. Eur. J. Neurosci. 27, 2871-2884.

Zwicker, J. D., Rajani, V., Hahn, L. B., and Funk, G. D. (2011). Purinergic modulation of preBötzinger complex inspiratory rhythm in rodents: the interaction between ATP and adenosine. J. Physiol. (Lond.) 589, 4583-4600.

Conflict of Interest Statement: The author declares that the research was conducted in the absence of any commercial or financial relationships that could be construed as a potential conflict of interest.

Received: 22 March 2012; accepted: 19 June 2012; published online: 20 July 2012. Citation: Peña-Ortega F (2012) Tonic neuromodulation of the inspiratory rhythm generator. Front. Physio. 3:253. doi: 10.3389/fphys.2012.00253

This article was submitted to Frontiers in Membrane Physiology and Biophysics, a specialty of Frontiers in Physiology. Copyright (c) 2012 Peña-Ortega. This is an open-access article distributed under the terms of the Creative Commons Attribution License, which permits use, distribution and reproduction in other forums, provided the original authors and source are credited and subject to any copyright notices concerning any third-party graphics etc. 